\title{
Extracting Safe Thread Schedules from Incomplete Model Checking Results
}

\author{
Patrick Metzler $^{1(\bowtie)}$, Neeraj Suri ${ }^{1}$, and Georg Weissenbacher ${ }^{2}$ \\ 1 Technische Universität Darmstadt, Darmstadt, Germany \\ patrick.metzler@posteo.net, suri@cs.tu-darmstadt.de \\ 2 TU Wien, Vienna, Austria \\ georg. weissenbacher@tuwien.ac.at
}

\begin{abstract}
Model checkers frequently fail to completely verify a concurrent program, even if partial-order reduction is applied. The verification engineer is left in doubt whether the program is safe and the effort towards verifying the program is wasted.

We present a technique that uses the results of such incomplete verification attempts to construct a (fair) scheduler that allows the safe execution of the partially verified concurrent program. This scheduler restricts the execution to schedules that have been proven safe (and prevents executions that were found to be erroneous). We evaluate the performance of our technique and show how it can be improved using partial-order reduction. While constraining the scheduler results in a considerable performance penalty in general, we show that in some cases our approach — somewhat surprisingly — even leads to faster executions.
\end{abstract}

\section{Introduction}

Automated verification of concurrent programs is inherently difficult because of exponentially large state spaces [38]. State space reductions such as partialorder reduction (POR) $[10,16,17]$ allow a model checker to focus on a subset of all reachable states while the verification result is valid for all reachable states. However, even reduced state spaces may be intractably large [17] and corresponding programs infeasible to (automatically) verify, requiring manual intervention.

We propose a novel model checking approach for safety verification of potentially non-terminating programs with a bounded number of threads, nondeterministic scheduling, and shared memory. Our approach iteratively generates incomplete verification results (IVRs) to prove the safety of a program under a (semi-)deterministic scheduler. The scheduling constraints induced by an IVR

P. Metzler-Supported by the German Academic Exchange Service (DAAD).

N. Suri-Research supported in part by H2020-SU-ICT-2018-2 CONCORDIA GA 830927 and BMBF-Hessen TUD CRISP.

G. Weissenbacher-Supported by the Vienna Science and Technology Fund (WWTF) through grant VRG11-005 and the Austrian Science Fund (FWF) via the Austrian National Research Network S11403-N23 (RiSE).

(C) Springer Nature Switzerland AG 2019

F. Biondi et al. (Eds.): SPIN 2019, LNCS 11636, pp. 153-171, 2019.

https://doi.org/10.1007/978-3-030-30923-7_9 
can be enforced by iteratively relaxed scheduling [29], a technique to enforce finegrained orderings of concurrent memory events. When the scheduling constraints of an IVR are enforced, all executions (under all non-deterministic inputs) are safe, even if the underlying (operating system) scheduler is non-deterministic. Thereby, the program can be executed safely before a (potentially infeasible) complete verification result is available. Executions can still exploit concurrency and the number of memory accesses that are executed concurrently may even be increased. As the model checking problem is eased, additional programs become tractable. Furthermore, IVRs can be used to safely execute unsafe programs which are safe under at least one scheduler. E.g., instead of programming synchronization explicitly, our model checking algorithm can be used to synthesize synchronization so that all executions are safe.

We use the producer-consumer example from Fig. 1 to explain our approach. The verifier analyses an initial schedule, e.g., where thread $T_{1}$ and $T_{2}$ produce and consume in turns, and emits an IVR $\mathcal{R}_{1}$, guaranteeing safe executions under this schedule. With its second IVR, the verifier might verify the correctness of producing two items in a row and the scheduling constraints can be relaxed accordingly. When the verifier hits an unsafe execution (the

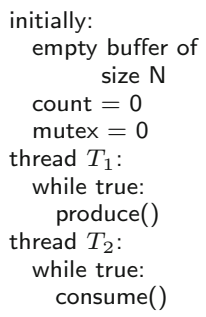

Fig. 1. Producer-consumer problem with bug

consumer produces an underflow), it emits an unsafe IVR for debugging. If the verifier accomplishes to analyze all possible executions of the program, it will report the final result partially safe, as the program can be used safely under all inputs but unsafe executions exist. Had there been no unsafe or safe IVR, the final result would be safe or unsafe, respectively.

This paper shows how to instantiate our approach by answering these questions: 1. Which state space abstractions are suitable for iterative model checking? The abstraction should be able to represent non-terminating executions and facilitate the extraction of schedules. 2. How to formalize and represent suitable IVRs? IVRs should be as small as possible in order to allow short iterations, while they must be large enough to guarantee fully functional executions under all possible program inputs. More precisely, for every possible program input, an IVR must cover a program execution. 3. What are suitable model checking algorithms that can be adapted to produce IVRs? A suitable algorithm should easily allow to select schedules for exploration.

\section{Incomplete Verification Results}

\subsection{Basic Definitions}

A program $P$ comprises a set $S$ of states (including a distinct initial state) and a finite set $\mathscr{T}$ of threads. Each state $s \in S$ maps program counters and variables to values. We use $\mathfrak{l}(s)$ to denote the program location of a state $s$, which comprises 
a local location $\mathfrak{l}_{T}(s)$ for each thread $T \in \mathscr{T}$. W.l.o.g. we assume the existence of a single error location that is only reachable if the program $P$ is not safe.

A state formula $\phi$ is a predicate over the program variables encoding all states $s$ in which $\phi(s)$ evaluates to true. A transition relation $R$ relates states $s$ and their successor states $s^{\prime}$. Each tread $T$ is partitioned into local transitions $R_{\mathfrak{l}, \mathfrak{l}^{\prime}}$ such that $\mathfrak{l}=\mathfrak{l}_{T}(s)$ and $\mathfrak{l}^{\prime}=\mathfrak{l}_{T}\left(s^{\prime}\right)$ for all $s, s^{\prime}$ satisfying $R_{\mathfrak{l}, \mathfrak{l}^{\prime}}\left(s, s^{\prime}\right)$ and $R_{\mathfrak{l}, \mathfrak{l}^{\prime}}$ leaves the program locations and variables of other threads unchanged. We use $\operatorname{Guard}(R)$ to denote a predicate encoding $\exists s^{\prime} . R\left(s, s^{\prime}\right)$, e.g., $\operatorname{Guard}\left(R_{13,14}\right)$ is (count $<\mathrm{N}$ ) for the transition from location 13 to 14 in Fig. 1. We say that $R_{\mathfrak{l}, \mathfrak{I}^{\prime}}$ (or $T$, respectively) is active at location $\mathfrak{l}$ and enabled in a state $s$ iff $\mathfrak{l}(s)=\mathfrak{l}$ and $s$ satisfies $\operatorname{Guard}(R)$. Multiple transitions of a thread $T$ at a location can be active, but we allow only one transition $R$ to be enabled at a given state and define $\operatorname{enabled}_{T}(s):=\{R\}$ if $R$ exists and $\operatorname{enabled}_{T}(s):=\emptyset$ otherwise.

If there exist states $s$ for which no transition of a thread $T$ is enabled (e.g., in line 12 in Fig. 1), $T$ may block. We assume that such locations $\mathfrak{l}_{T}(s)$ are (conservatively) marked by may-block $\left(\mathfrak{l}_{T}(s)\right)$.

An execution is a sequence $s_{0}, T_{1}, s_{1}, \ldots$, where $s_{0}$ is the initial state and the states $s_{i}$ and $s_{i+1}$ in every adjacent triple $\left(s_{i}, T_{i}, s_{i+1}\right)$ are related by the transition relation of $T_{i}$. An execution that does not reach the error location is safe. A deadlock is a state $s$ in which no transitions are enabled. W.l.o.g. we assume that all finite executions correspond to deadlocks and are undesirable; intentionally terminating executions can be modelled using terminal locations with self-loops.

An execution $\tau$ is (strongly) fair if every thread $T_{i}$ enabled infinitely often in $\tau$ is also scheduled infinitely often [5]. We assume that fairness is desirable and enforce it by our algorithm presented in Sect. 3. Other notions of fairness such as weak fairness can be enforced analogously.

Non-determinism can arise both through scheduling and non-deterministic transitions. A scheduler can resolve the former kind of non-determinism.

Definition 1 (scheduler). A scheduler $\zeta:(S \times \mathscr{T})^{*} \times S \rightarrow \mathscr{T}$ of a program $P$ is a function that takes an execution prefix $s_{0}, T_{1}, \ldots, T_{n}, s_{n}$ and selects a thread that is enabled at $s_{n}$, if such a thread exists. A scheduler $\zeta$ is deadlock-free (fair, respectively) if all executions possible under $\zeta$ are deadlock-free (fair).

A scheduler for the program of Fig. 1, for instance, must select $T_{1}$ rather than $T_{2}$ for the prefix $s_{\text {init }}, T_{1}, s_{1}, T_{1}, s_{2}, T_{1}, s_{3}, T_{2}, s_{4}, T_{2}, s_{5}$, since at that point the lock is held by $T_{1}$ and enabled $_{T_{2}}\left(s_{5}\right)=\emptyset$.

Non-deterministic transitions are the second source of non-determinism. If $R_{\mathfrak{l}, l^{\prime}}$ of thread $T$ allows multiple successor states for a state $s$, we presume the existence of input symbols $X$ such that each $\iota \in X$ determines a unique successor state $s^{\prime}$ by selecting an $R_{\mathfrak{l}, \mathfrak{l}^{\prime}}^{\iota} \subseteq R_{\mathfrak{l}, \mathfrak{l}^{\prime}}$ with $R_{\mathfrak{l}, \mathfrak{l}^{\prime}}^{\iota}\left(s, s^{\prime}\right)$.

Definition 2 (input). An input is a function $\chi:(S \times \mathscr{T})^{*} \rightarrow X$, which chooses an input symbol depending on the current execution prefix. 
In conjunction, an input and a scheduler render a program completely deterministic: the input $\chi$ and scheduler $\zeta$ select a transition in each step such that each adjacent triple $\left(s_{i}, T_{i+1}, s_{i+1}\right)$ is uniquely determined.

For Partial Order Reduction (POR), we assume that a symmetric independence relation $\|$ on transitions of different threads is given, which induces an equivalence relation on executions. Two transitions $R_{1}$ and $R_{2}$ are only independent if they are from distinct threads, they are commutative at states where both $R_{1}$ and $R_{2}$ are enabled, and executing $R_{1}$ does neither enable nor disable $R_{2}$. We write $R_{1} \nVdash R_{2}$ if $R_{1}$ and $R_{2}$ are not independent.

\subsection{Requirements on Incomplete Verification Results}

Our goal is to ease the verification task by producing incomplete verification results (IVRs) which prove the program safety under reduced non-determinism, i.e., only for a certain scheduler. We only allow "legitimate" restrictions of the scheduler that do not introduce deadlocks or exclude threads. Inputs must not be restricted, since this might reduce functionality and result in unhandled inputs.

Hence, we define an IVR to be a function $\mathcal{R}$ that maps execution prefixes to sets of threads, representing scheduling constraints. An IVR for the program from Fig. 1, for instance, may output $\left\{T_{1}\right\}$ in states with an empty buffer, meaning that only thread $T_{1}$ may be scheduled here, and $\left\{T_{2}\right\}$ otherwise, so that an item is produced if and only if the buffer is empty. A scheduler $\zeta_{\mathcal{R}}$ enforces (the scheduling constraints of) an IVR $\mathcal{R}$ if $\zeta_{\mathcal{R}}(\tau) \in \mathcal{R}(\tau)$ for all execution prefixes $\tau$. IVR $\mathcal{R}$ permits all executions possible under a scheduler that enforces $\mathcal{R}$.

The remainder of this subsection discusses the requirements on useful IVRs. We define safe, realizable, deadlock-free, fairness-admitting, and fair IVRs. In the following subsection, we instantiate IVRs with abstract reachability trees (ARTs).

Safety. An IVR $\mathcal{R}$ can either expose a bug in a program or guarantee that all permitted executions are safe. Here, we are only concerned with the latter case. An IVR $\mathcal{R}$ is safe if all executions permitted by $\mathcal{R}$ are safe. An unsafe IVR permits an unsafe execution and is called a counterexample.

Completeness. To reduce the work for the model checker, a safe IVR $\mathcal{R}$ should ideally have to prove the correctness of as few executions as possible. At the same time, it should cover sufficiently many executions so that the program can be used without functional restrictions. For instance, the $\operatorname{IVR} \mathcal{R}(\tau):=\emptyset$, for all $\tau$, is safe but not useful, as it does not permit any execution. Consequently, $\mathcal{R}$ should permit at least one enabled transition, in all non-deadlock states, which is done by realizable IVRs: an IVR $\mathcal{R}$ is realizable if at least one scheduler that enforces $\mathcal{R}$ exists. Furthermore, an IVR should never introduce a deadlock: an IVR $\mathcal{R}$ is deadlock-free if all schedulers that enforce $\mathcal{R}$ are deadlock-free.

Fairness. In general, we deem only fair executions desirable. The $\operatorname{IVR} \mathcal{R}(\tau):=$ $\left\{T_{1}\right\}$, for instance, is deadlock-free for the program of Fig. 1 but useless, as no item is consumed. A deadlock-free IVR admits fairness if there exists a fair scheduler enforcing $\mathcal{R}$ (i.e., a fair execution of the program is possible). 
If a scheduler permits both fair and unfair executions, it might be difficult to guarantee fairness at runtime. In such cases, a fair IVR can be used: A deadlockfree IVR $\mathcal{R}$ is fair if all schedulers enforcing $\mathcal{R}$ are fair.

\subsection{Abstract Reachability Trees as Incomplete Verification Results}

In this subsection, we instantiate the notion of IVRs using abstract reachability trees (ARTs), which underly a range of software model checking tools $[9,21,23,28]$ and have recently been used for concurrent programs [39]. Due to the explicit representation of scheduling choices from the beginning of an execution up to an (abstract) state, ARTs are well-suited to represent IVRs. Model checking algorithms based on ARTs perform a path-wise exploration of program executions and represent the current state of the exploration using a tree in which each node $v$ corresponds to a set of states at a program location $\mathfrak{l}(v)$. These states, represented by a predicate $\phi(v)$, (safely) over-approximate the states reachable via the program path from the root of the ART $(\epsilon)$ to $v$. Edges expanded at $v$ correspond to transitions starting at $\mathfrak{l}(v)$. A node $w$ may cover $v$ (written $v \triangleright w$ ) if the states at $w$ include all states at $v(\phi(v) \Rightarrow \phi(w))$; in this cases, $v$ is covered (covered $(v))$ and its successors need not be further explored. (Intuitively, executions reaching $v$ are continued from $w$.) Formally, an ART is defined as follows:

Definition 3 (abstract reachability tree [28,39]). An abstract reachability tree $(A R T)$ is a tuple $\mathscr{A}=(V, \epsilon, \rightarrow, \triangleright)$, where $(V, \rightarrow)$ is a finite tree with root $\epsilon \in V$ and $\triangleright \subseteq V \times V$ is a covering relation. Nodes $v$ are labeled with global control locations and state formulas, written $\mathfrak{l}(v)$ and $\phi(v)$, respectively. Edges $(v, w) \in \rightarrow$ are labeled with a thread and a transition, written $v \stackrel{T, R}{\longrightarrow} w$.

Intuitively, an ART $\mathscr{A}$ is well-labeled [28] if $\mathscr{A}$ 's $\rightarrow$-edges represent the transitions of the program and edges $v \triangleright w$ indicate that all states modeled by node $v$ are also modeled by node $w$. Formally, $\mathscr{A}$ is well-labeled if for every edge $v \stackrel{T, R_{\mathrm{r}, \mathrm{l}^{\prime}}}{\longrightarrow} w$ in $\mathscr{A}$ we have that (i) $\phi(\epsilon)$ represents the initial state, (ii) $\phi(v)(s) \wedge R_{\mathfrak{l}, \mathfrak{l}^{\prime}}\left(s, s^{\prime}\right) \Rightarrow \phi(w)\left(s^{\prime}\right)$ and $\mathfrak{l}_{T}(v)=\mathfrak{l}$ and $\mathfrak{l}_{T}(w)=\mathfrak{l}^{\prime}$, and (iii) for every $v, w$ with $v \triangleright w, \phi(v) \Rightarrow \phi(w)$ and $\neg \operatorname{covered}(w)$.

An incomplete ART $\mathscr{A}_{p-c}$ for the producer-consumer problem of Fig. 1 is shown on the right. Nodes show the state formulas and edges are labeled with the thread and statement corresponding to the transition.

ART-Induced Schedulers. A well-labeled ART $\mathscr{A}$ directly corresponds to an IVR $\mathcal{R}_{\mathscr{A}}$ that simulates an execution by traversing $\mathscr{A}$. We define $\mathcal{R}_{\mathscr{A}}$ as follows:

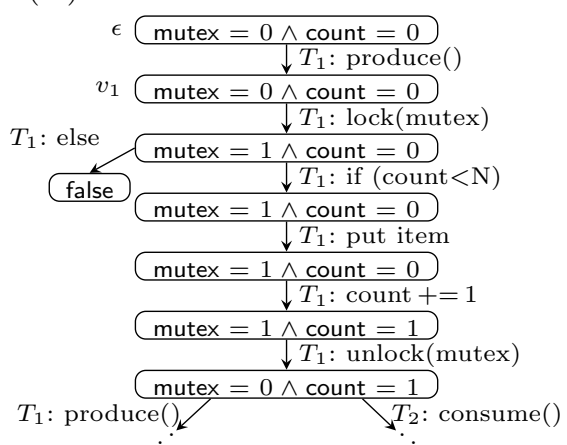
Let $\tau=s_{0}, T_{1}, s_{1}, \ldots, s_{n}$ be an execution 
prefix. If $\mathscr{A}$ contains no path that corresponds to $\tau, \mathcal{R}_{\mathscr{A}}$ leaves the schedules for this execution unconstrained. Otherwise, let $v_{n}$ be the last node of the path in $\mathscr{A}$ that corresponds to $\tau$. $\mathcal{R}_{\mathscr{A}}$ permits exactly those threads that are expanded at $v_{n}$ (or at $w$ if $v_{n}$ is covered by some node $w$ ). E.g., the execution prefix $\tau=s_{0}, T_{1}, s_{1}$ corresponds to the path from $\epsilon$ to $v_{1}$ in $\mathscr{A}_{p-c}$. As only $T_{1}$ is expanded at $v_{1}, \mathcal{R}_{\mathscr{A} p-c}$ allows only $\left\{T_{1}\right\}$ after $\tau$.

Safety. An ART is safe if whenever $\mathfrak{l}_{T}(v)$ is the error location then $\phi(v)=$ false. As only safe executions may correspond to a path in a safe ART (cf. Theorem 3.3 of [39]), $\mathcal{R}_{\mathscr{A}}$ is a safe IVR.

Completeness. In order to derive a deadlock-free IVR from a well-labeled ART $\mathscr{A}$, we have to fully expand at least one thread $T$ at each node $v$ that represents reachable states (where $T$ is fully expanded at $v$ if $v$ has an outgoing edge for every active transition of $T$ at $\mathfrak{l}_{T}(v)$ ). However, there may exist reachable states $s$ represented by $\phi(v)$ for which no action of $T$ is enabled (i.e., $\operatorname{enabled}_{T}(s)=\emptyset$ ). If $T$ is the only thread expanded at $v, \mathcal{R}_{\mathscr{A}}$ is not realizable. This situation can arise for locations $\mathfrak{l}$ at which $T$ may block (marked with may-block $\left(\mathfrak{l}_{T}\right)$ ).

Consequently, whenever may-block $\left(\mathfrak{l}_{T}(v)\right)$ in a deadlock-free ART $\mathscr{A}$, we require that $\phi(v)$ is strong enough to entail that the transitions $R$ of $T$ expanded at $v$ (or at the node covering $v$, respectively) are enabled (i.e., $\phi(v) \Rightarrow \operatorname{Guard}(R))$. For instance, $\phi\left(v_{1}\right)$ in the ART shown above proves the enabledness of $T_{1}$ at $v_{1}$, as $\phi\left(v_{1}\right) \Rightarrow$ mutex $=0$ and lock(mutex) is enabled if mutex $=0$.

Lemma 1. If an ART $\mathscr{A}$ is deadlock-free, $\mathcal{R}_{\mathscr{A}}$ is a deadlock-free IVR.

Fairness. IVRs derived from deadlock-free ARTs do not necessarily admit fairness if the underlying ART contains cycles (across $\triangleright$ and $\rightarrow$ edges) that represent unfair executions. In order to make sure a deadlock-free ART admits fairness we implement a scheduler that allows $\mathscr{A}$ to schedule each thread infinitely often (whenever it is enabled infinitely often) by requiring that every $(\triangleright \cup \rightarrow$ )-cycle is "fair", defined as follows.

Definition 4 (ART admitting fairness). A deadlock-free $A R T \mathscr{A}=(V, \epsilon, \rightarrow$ $, \triangleright)$ admits fairness if every $(\triangleright \cup \rightarrow)$-cycle contains, for every thread $T$ that is enabled at a node of the cycle, a node $v$ such that $T$ is expanded at $v$.

Lemma 2. If an ART $\mathscr{A}$ admits fairness, $\mathcal{R}_{\mathscr{A}}$ is an IVR that admits fairness.

Note that the expansion of a thread $T$ at a node in a cycle does not guarantee that the transition is part of the cycle. A slight modification of the fairness condition for ARTs leads to a sufficient condition for ARTs as fair IVRs, as the following definition and lemma show. The difference in the fairness condition is that all enabled threads are expanded within each $(\triangleright \cup \rightarrow)$-cycle $c$, which we denote by fair $(c)$. The $(\triangleright \cup \rightarrow)$ cycle shown on the right, for instance, is fair.

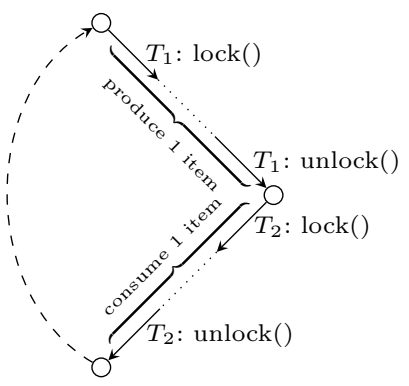




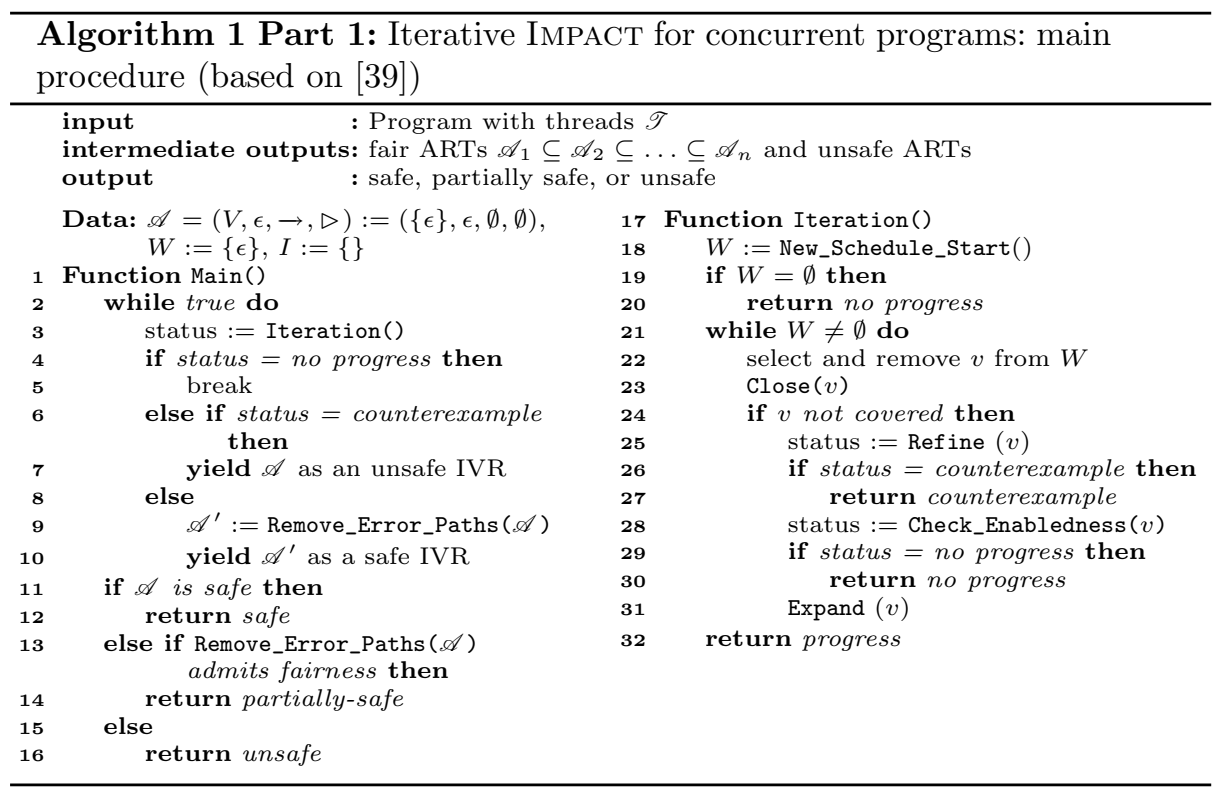

Definition 5 (fair ART). A deadlock-free $A R T \mathscr{A}=(V, \epsilon, \rightarrow, \triangleright)$ is fair if fair $(c)$ holds for every $(\triangleright \cup \rightarrow)$-cycle $c$.

Lemma 3 (fairness). For all fair ARTs $\mathscr{A}, \mathcal{R}_{\mathscr{A}}$ is a fair IVR.

Given an ART $\mathscr{A}$ that admits fairness, one can generate a fair ART $\mathscr{A}^{\prime}$ such that $\mathcal{R}_{\mathscr{A}}$ permits all executions permitted by $\mathcal{R}_{\mathscr{A}}$.

\section{$3 \quad$ Iterative Model Checking}

A suitable algorithm for our framework must generate fair IVRs. We use model checking based on ARTs (cf. Sect. 2.3), which allows us to check infinite executions and explicitly represent scheduling. Nevertheless, other program analysis techniques such as symbolic execution are also suitable to generate IVRs. In particular, our algorithm (Algorithm 1 parts 1 and 2) constitutes an iterative extension of the IMPACT algorithm [28] for concurrent programs [39]. We chose IMPACT as a base for our algorithm because it has an available implementation for multi-threaded programs, which we use to evaluate our approach in Sect. 5.

IMPACT generates an ART by path-wise unwinding the transitions of a program. Once an error location is reached at a node $v$, IMPACT checks whether the path $\pi$ from the ART's root to $v$ corresponds to a feasible execution. If this is the case, a property violation is reported; otherwise, the node labeling is strengthened via interpolation. Thereby, a well-labeled ART is maintained. Once the ART is complete, its node labeling provides a safety proof for the program. 


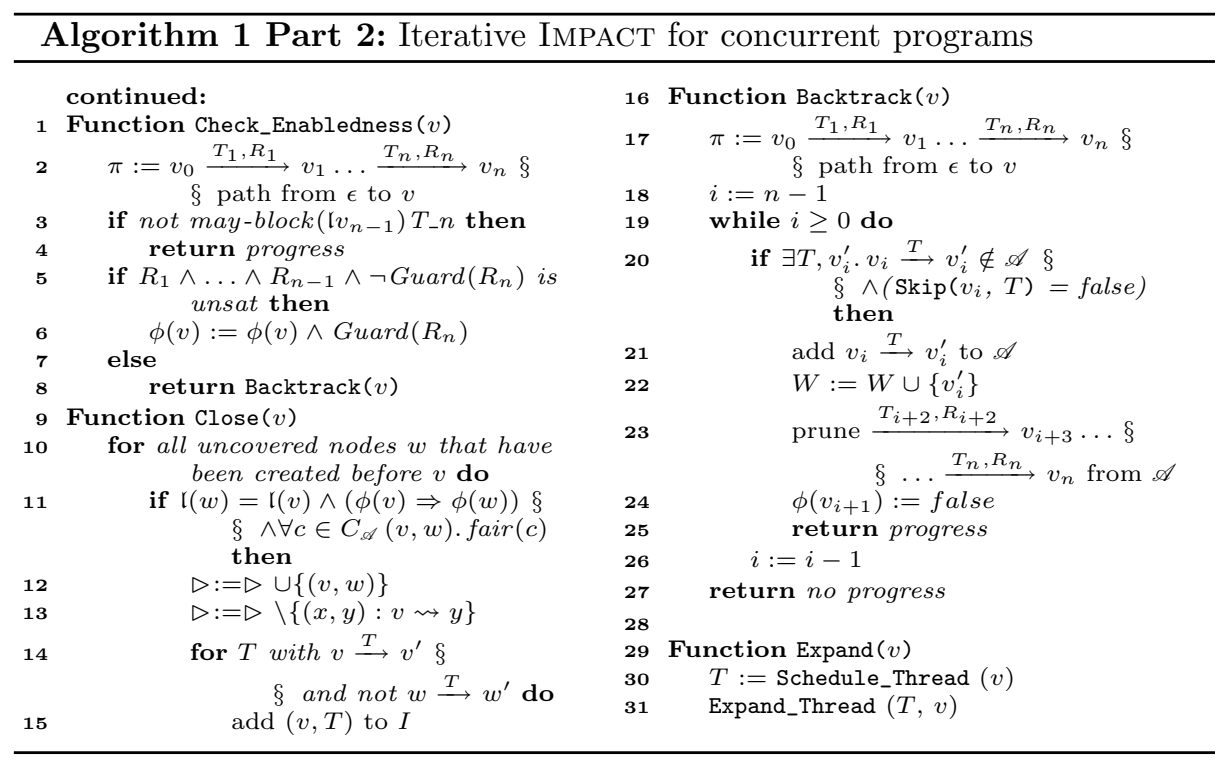

In each iteration, our extended algorithm yields an IVR which is either unsafe (a counterexample) or fair (can be used as scheduling constraints). If the algorithm terminates, it outputs "safe", "partially safe", or "unsafe", depending on whether the program is safe under all, some, or no schedulers. Procedure Main() repeatedly calls Iteration() (line 3), which, intuitively, corresponds to an execution of the original algorithm of [39] under a deterministic scheduler. Iteration() (potentially) extends the ART $\mathscr{A}$. If no progress is made ( $\mathscr{A}$ is unchanged), the algorithm terminates (lines 12, 14, and 16). Otherwise, an intermediate output is yielded: either $\mathscr{A}$ as an intermediate output (line 7) or $\mathscr{A}$ with all previously found counterexamples removed, i.e., the largest fair ART that is a subgraph of $\mathscr{A}$, denoted by Remove_Error_Paths().

Iteration() maintains a work list $W$ of nodes $v$ to be explored via Close $(v)$ (Algorithm 1 part 2), which tries to find (as in [39]) a node that covers $v$. In addition to the covering check of [39], we check fairness, where $C_{\mathscr{A}}(v, w)$ denotes all cycles that would be closed by adding the edge $v \triangleright w$ (line 11 of Algorithm 1 part 2). If such a node $w$ is found, any thread $T$ that is expanded at $v$ but not at $w$ (line 14 of Algorithm 1 part 2) must not be skipped at $w$ by POR. Instead of expanding $T$ instantaneously at $w$ (as in [39]), which would explore another schedule, $T$ is added to the set $I$ so that it can be explored in a subsequent iteration. If no covering node for $v$ is found, $v$ is refined, which returns counterexample if $v$ has a feasible error path (line 25). Otherwise (line 28), Check_Enabledness() (Algorithm 1 part 2) performs a deadlock check by testing whether the last action that leads to $v$ is enabled in all states represented by the predecessor node. If not, deadlock-freedom is not guaranteed and Backtrack() tries to find a substitute node where exploration can continue. 

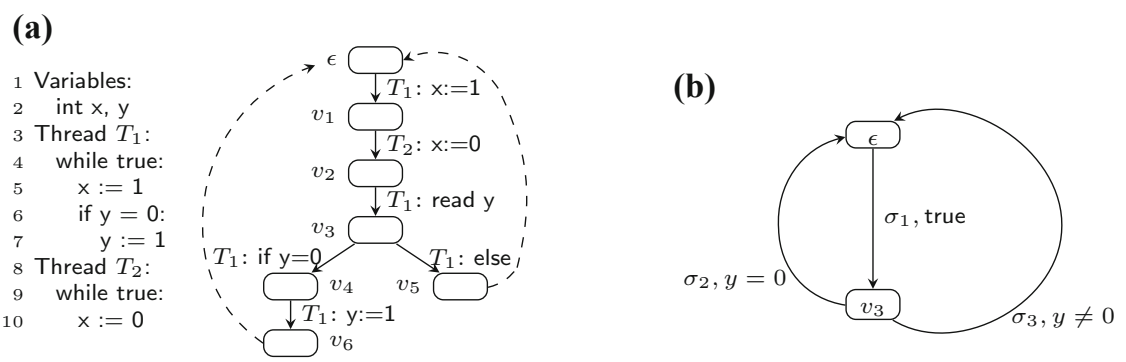

Fig. 2. (a) Section paths. (b) A program schedule

The deterministic scheduler of Iteration() is controlled by New_Schedule_Start() and Schedule_Thread(). The former selects a set of initial nodes for the exploration (line 18 of Algorithm 1 part 1); the latter decides which thread to expand at a given node (line 30 of Algorithm 1 part 2). We use a simple heuristic that selects the first (in breadth-first order) node which is not yet fully expanded and use a round-robin scheduler for Schedule_Thread that switches to the next thread once a back jump occurs (e.g., the end of a loop body is reached). Additionally, Schedule_Thread returns only threads that are necessary to expand at the given node after POR (cf. Skip() [39]). More elaborate heuristics are conceivable but out of the scope of this paper.

The correctness of Algorithm 1 w.r.t. safety follows from the correctness of [28] and [39]. Additionally, Algorithm 1 is also fair:

Lemma 4 (fairness of Algorithm 1). Any safe ART $\mathscr{A}$ generated by Algorithm 1 is fair.

\section{Partial-Order Reduction}

A naive enforcement of the context switches at the relevant nodes of a safe IVR $\mathcal{R}_{\mathscr{A}}$ would result in a strictly sequential execution of the transitions, foiling any benefits of concurrency. To enable parallel executions, we introduce program schedules that relax the scheduling constraints by means of partial-order reduction (POR). Note that this application of POR concerns the enforcement of scheduling constraints and occurs in addition to POR applied by our model checking algorithm when constructing an ART (cf. Sect. 3). Nevertheless, dependency information that is used for POR during model checking can be reused so that redundant computations are avoided.

The goal is to permit the parallel execution of independent transitions (in different threads) whose order does not affect the outcome of the execution represented by $\mathscr{A}$ (i.e., the resulting traces are Mazurkiewicz-equivalent). Using traditional POR to construct such scheduling constraints poses two challenges: 1. Executions may be infinite, but we need a finite representation of scheduling constraints. 2. The control flow of an execution may be unpredictable, i.e., it 
is a priori unclear which scheduling constraints will apply. We solve issue 1 by partitioning ARTs into sections and associate a finite schedule with every section. To address issue 2 , we require that sections do not contain branchings (control flow and non-deterministic transitions).

Consider the program and corresponding ART in Fig. 2a. The if-statement of $T_{1}$ is modeled as a separate read transition followed by a branching at node $v_{3}$. We define three section paths $\pi_{1}:=\epsilon \rightarrow v_{1} \rightarrow v_{2} \rightarrow v_{3}, \pi_{2}:=v_{3} \rightarrow v_{4} \rightarrow$ $v_{6} \rightarrow \epsilon$, and $\pi_{3}:=v_{3} \rightarrow v_{5} \rightarrow \epsilon$. After $\pi_{1}$ has been executed, a scheduler can distinguish the cases $y=0$ and $y \neq 0$ and schedule $\pi_{2}$ or $\pi_{3}$ accordingly.

Formally, a section path $v_{1} \stackrel{R_{1}}{\longrightarrow} \ldots \stackrel{R_{n}}{\longrightarrow} v_{n+1}$ corresponds to a branching-free path in an ART whose first transition may be guarded. A section path follows $\rightarrow \mathscr{A}$ edges, skipping covering edges $\triangleright$. The section schedule of a section path describes the Mazurkiewicz equivalence class of the contained transitions and is defined as the smallest partial order $\sigma=\left(V_{\sigma}, \rightarrow_{\sigma}\right)$ such that $V_{\sigma}=\left\{e_{1}, \ldots, e_{n}\right\}$ and $\rightarrow \sigma \supseteq\left\{\left(e_{i}, e_{j}\right): i<j \wedge R_{i} \nVdash R_{j}\right\}$, where $e_{i}, 1 \leq i \leq n$ is the occurrence of transition $R_{i}$ at position $i$. The section schedule of $\pi_{1}$ is $\left(\left\{e_{1}, e_{2}, e_{3}\right\},\left\{\left(e_{1}, e_{2}\right),\left(e_{1}, e_{3}\right)\right\}\right)$ with $e_{1} \triangleq T_{1}:$ x:=1, $e_{2} \triangleq T_{2}:$ x:=0, and $e_{3} \triangleq T_{1}:$ read $\mathrm{y}$.

A program schedule $\Sigma$ comprises several section schedules. $\Sigma$ is a labeled graph $\left(V_{\Sigma}, \rightarrow_{\Sigma}\right)$. Each node $v \in V_{\Sigma}$ is the start of a section path $\pi$ in $\mathscr{A}$. Each edge is labeled with the section schedule of $\pi$ and the guard $\operatorname{Guard}(R)$ of the first transition $R$ in $\pi$. As $\mathscr{A}$ is deadlock-free, there exists a thread $T$ which is fully expanded at $v$ in $\mathscr{A}$ and we require that $\Sigma$ likewise has outgoing edges at $v$ labeled with $T$ for each transition of $T$ at $v$. Figure $2 \mathrm{~b}$ shows a program schedule for our example program.

A scheduler can enforce the scheduling constraints of a program schedule by picking a section schedule that matches the current execution prefix and scheduling an event whose predecessors (according to the section schedule) have already been executed. Hence, all independent events in a section can be executed concurrently without synchronization. All events of a section schedule have to appear before the first event of the next section schedule, so that the states reached between sections correspond to nodes of the program schedule.

A program schedule of an ART $\mathscr{A}$ that admits fairness permits exactly those executions that correspond to a path in $\mathscr{A}$ (modulo Mazurkiewicz equivalence). In particular, as Mazurkiewicz equivalence preserves safety properties [17], only safe executions are permitted.

Lemma 5 (correctness). Let $\mathscr{A}$ be an ART that admits fairness and $\Sigma$ a program schedule for $\mathscr{A}$. All program executions induced by $\Sigma$ are equivalent to an execution that corresponds to a path in $\mathscr{A}$.

\section{Evaluation}

In five case studies, we evaluate our iterative model checking algorithm and scheduling based on IVRs. We use the IMPARA model checker [39], as it is the only 
available implementation of model checking for non-terminating, multi-threaded programs based on a forward analysis on ARTs we have found. IMPARA uses lazy abstraction with interpolants based on weakest preconditions. We extend the tool by implementing our algorithm presented in Sect. 3. IMPARA accepts $\mathrm{C}$ programs as inputs, however, some language features are not supported and we have rewritten programs accordingly. ${ }^{1}$ We refer to the (non-iterative) IMPARA tool as IMPARA-C (for complete verification) and to our extension of Impara with iterative model checking as IMPARA-IMC.

Based on the ARTs constructed by IMPARA, program schedules are generated automatically and encoded as vector clocks. We instrument the benchmark programs with a call-back to a specially designed user space scheduler directly before and after each access to a global variable. The result is a multi-threaded program that executes concurrent memory accesses according to a given program schedule. All experiments have been executed on a 4-core Intel Core i5-6500 CPU at $3.2 \mathrm{GHz}$. We report median values averaged over five runs.

\subsection{Infeasible Complete Verification}

Even for a moderate number of threads, complete verification, i.e., verification of a program under all possible schedules and inputs, may be infeasible. In particular, IMPARA$\mathrm{C}$ times out (after $72 \mathrm{~h}$ ) on a corrected variant of the producer consumer problem (Fig. 1) with four producers and four consumers. IMPARA-IMC produces the first IVR $\mathcal{R}_{1}$ after $4: 29: 53 \mathrm{~h}$. A simplification of $\mathcal{R}_{1}$ is depicted on the right; it covers all executions in which the threads appear

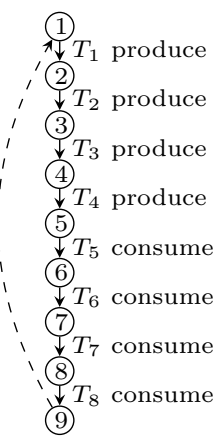
to execute their loop bodies atomically in the order $T_{1}, T_{2}, \ldots, T_{8}$. While the main bottleneck for IMPARA-C is state explosion and finding many coverings for different schedules, we observe that the main issue to produce $\mathcal{R}_{1}$ is to find a single covering that comprises all threads, i.e., to find a fair cycle.

The subsequent IVRs $\mathcal{R}_{2}, \ldots, \mathcal{R}_{8}$ are found much faster than the first IVR, after $19: 31,12: 3,6: 13,28: 0,9: 25,8: 27$, and 8:40 min. We stop the model checker after eight IVRs. According to our implementation of New_Schedule Start() in Algorithm 1, IVR $\mathcal{R}_{i}$ permits, in addition to all executions permitted by $\mathcal{R}_{i-1}$, those executions in which the threads appear in the order $T_{i}, T_{1}, \ldots, T_{i-1}, T_{i+1}, \ldots, T_{8}$. Hence, $\mathcal{R}_{8}$ gives the scheduler more freedom than $\mathcal{R}_{1}$, which may result in a better execution performance, e.g., because a producer which has its item available earlier does not have to wait for all previous producers.

${ }^{1}$ E.g., Pthread mutexes, some uses of the address-of operator, and reuse of the same function by several threads are not supported. We solve these issues by rewriting our benchmark programs so that IMPARA handles them correctly and their intuitive semantics is not changed. We will publish our modifications to IMPARA, including two bug fixes. 


\subsection{Declarative Synchronization}

Figure 3 shows an extension of a benchmark used in [15], which is a simplified extract of the multi-threaded Frangipani file system. The program uses a timevarying mutex: depending on the current value of the busy bit, a disk block is protected by m_busy or m_inode. We want to evaluate whether we can use IMPARAIMC to generate safe program schedules even if all mutexes are (intentionally) removed from the program.

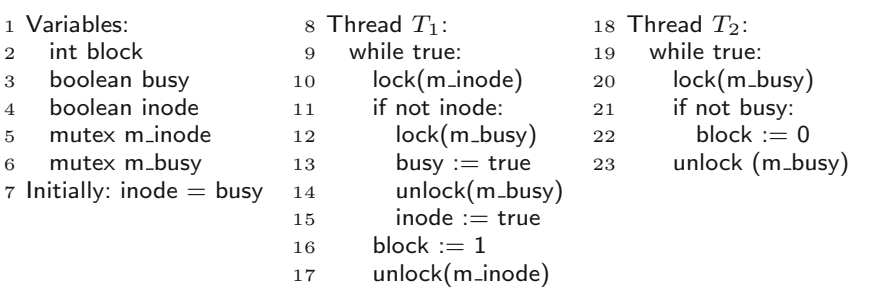

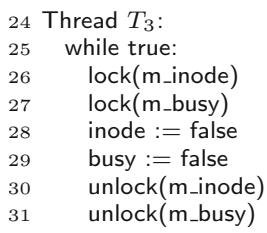

Fig. 3. The file system benchmark

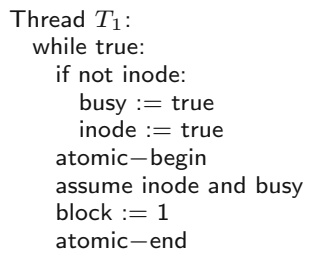

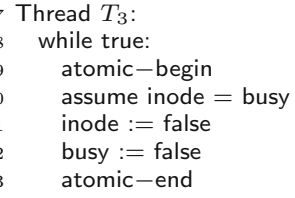

Fig. 4. The file system benchmark with synchronization constraints in assume statements

For this purpose, we use a variant of the file system benchmark where all mutexes are removed and synchronization constraints are declared as assume statements, shown in Fig. 4. It is sufficient to assure for $T_{1}$ that the block is written only if it is allocated, i.e., both inode and busy are true. For $T_{2}$, it is sufficient to assure that the block is only reset if it is not busy, i.e., busy = false. Finally, for $T_{3}$, it is necessary to assure that the block is deallocated only if it is already deallocated or fully allocated, i.e., inode = busy.

Running IMPARA-IMC on the file system benchmark without mutexes yields a first program schedule that schedules $T_{1}, T_{2}, T_{3}$ repeatedly in this order, according to our simple heuristic for an initial IVR. However, although all executions permitted by this schedule are fair, the if-

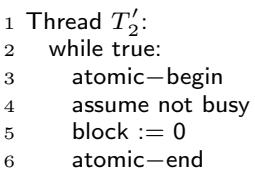


that the (omitted) else-branch of Thread $T_{2}$ is not useful. We encode this information by inserting else: assume false. After simplifying the code, we obtain $T_{2}^{\prime}$ as depicted on the right. For the updated code, IMPARA-IMC yields a first scheduler that schedules $T_{3}$ before $T_{2}$ before $T_{1}$, so that all threads perform useful work.

\subsection{Performance}

Table 1 shows the performance impact of enforcing IVRs on several correct programs. Each program is model-checked once until the first IVR (IMPARAIMC) and once completely (IMPARA-C). As a baseline, the program is run without schedule enforcement (unconstrained). The first IVR is enforced without (Opt0), and with optimizations (Opt1, Opt2). Opt1 applies POR and omits operations on synchronization objects (mutexes, barriers). ${ }^{2}$ Opt2 uses, in addition to Opt1, longer section schedules (by replicating a section eight times) and stronger partial-order reduction that identifies independent accesses to distinct indices of an array. Additionally, for the producer-consumer benchmark, we apply a compiler-like optimization, removing and reordering events to reduce the number of constraints. ${ }^{3}$ Both Opt1 and Opt2 enable the concurrent execution of more memory accesses, e.g., because the beginning of a critical section can already be executed before a thread arrives at a constrained access that has to wait. The schedules for each benchmark (Opt0-Opt2) are obtained from the first IVR. As all benchmarks use unbounded loops, we measure the execution time performance by counting useful (i.e., with a successful concurrent access such as a produced item) loop iterations and terminating the execution after $2 \mathrm{~s}$.

Table 1. Experimental results (to: timeout, rounded to full seconds). Performance is measured in number of useful (e.g., with a successful concurrent access such as a produced item) loop iterations within a time limit of $2 \mathrm{~s}$.

\begin{tabular}{|c|c|c|c|c|c|c|}
\hline \multirow[t]{2}{*}{ Benchmark } & \multicolumn{2}{|c|}{ Model checking } & \multicolumn{4}{|c|}{ Performance (higher is better) } \\
\hline & Time 1st IVR & IMPARA-C & Opt0 & Opt1 & Opt2 & Unconstr \\
\hline prod.-cons. 1p 1c & $2 \mathrm{~m} \mathrm{0s}$ & to $(72 \mathrm{~h})$ & 4864489 & 7466093 & 11370258 & 8199202 \\
\hline prod.-cons. $2 \mathrm{p} 2 \mathrm{c}$ & $23 \mathrm{~m} 47 \mathrm{~s}$ & to $(72 \mathrm{~h})$ & 3400187 & 5959041 & 8428598 & 11643208 \\
\hline prod.-cons. 4p 4c & $4 \mathrm{~h} 29 \mathrm{~m} 53 \mathrm{~s}$ & to $(72 \mathrm{~h})$ & 1327063 & 2576695 & 3676876 & 7210796 \\
\hline double lock $1 \mathrm{~ms}$ & $0 \mathrm{~s}$ & $0 \mathrm{~s}$ & 1845 & 1834 & 3217 & 1797 \\
\hline file system & $0 \mathrm{~s}$ & $0 \mathrm{~s}$ & 3667 & 4877035 & 6705672 & 23822129 \\
\hline barrier & $1 \mathrm{~s}$ & $4 \mathrm{~m} 14 \mathrm{~s}$ & 1238720 & 8285228 & 14586849 & 1077907 \\
\hline
\end{tabular}

We use the producer-consumer implementation (with correct synchronization and buffer size 1000) from SV-COMP [1] (stack_safe), modified with an

\footnotetext{
${ }^{2}$ As enforcing an IVR is redundant to synchronization over existing mutexes and barriers, omitting them is safe.

${ }^{3}$ Opt2 follows a general algorithm, however we do not automate our implementation of Opt2, as it would be a large effort to implement compiler optimizations. Our implementation of Opt1 is automated.
} 
unbounded loop and with 1, 2, and 4 producers and consumers. The double lock benchmark is a corrected version (lock operations in $T_{2}$ reversed) of the deadlock benchmark (Sect. 5.2), where the critical section is simulated by sleeping for $1 \mathrm{~ms}$; the uncorrected version reached a deadlock after only 172 loop iterations. The file system benchmark from SV-COMP (time_var_mutex_safe) is extended with a third thread and again with unbounded loops as in Sect. 5.4. The barrier benchmark uses two barriers to implement ring communication between threads.

As the model checking columns of Table 1 show, IMPARA-IMC finds the first IVR often much faster than or at least as fast as it takes IMPARA-C for complete model checking; it can produce an IVR even for our largest benchmarks, where IMPARA-C times out. For a buffer size of 5, IMPARA-C can verify the producer-consumer benchmark even with eight threads but again, IMPARA-IMC is considerably faster in finding the first IVR. Subsequent IVRs were generated considerably faster than the first IVR, which might be caused by caching of facts in the model checker.

Somewhat surprisingly, some benchmarks are slower when executed unconstrained. We conjecture that this is caused by more memory accesses being executed in parallel under Opt2. In all but one cases, Opt2 is considerably faster than Opt1, which is considerably faster than Opt0. The highest overhead is observed for the file system benchmark, where Opt2 is about 3.5 times slower than the unconstrained execution. We conjecture that the high overhead here stems from an unequal distribution of loop iterations among threads, when executed unconstrained: the loop body of $T_{2}$ was executed nearly 100 times more frequently than $T_{1}$, while it is shorter and probably faster. Opt0-Opt2 execute all threads nearly balanced. In addition to the Pthread barriers used in the barrier benchmark, we tried a variant with busy waiting barriers, where the unconstrained execution showed a performance of 13567135 , which is still slower than Opt2. When the buffer size of the producer-consumer benchmark with eight threads is reduced to 5 , the performance of unconstrained executions decreases to 3240136 compared to 3392111 with Opt2.

Even in repeated executions of the experiment, the unconstrained variant of double lock showed only "starving" executions in the sense that the second thread was never able to acquire the mutexes before the timeout of $2 \mathrm{~s}$. Hence, the constrained executions improve on the operating system scheduler in terms of a balanced execution of all threads.

In order to compare to the enforcement of input-covering schedules [7] (explained in Sect.6), we measure the overhead of our scheduler implementation on the pfscan benchmark used there. Pfscan is a parallel implementation of grep and uses 1 producer and 2 consumer threads to distribute tasks, consisting of reading and searching a file for a given query. As input, we use 8 files with $100 \mathrm{MB}$ of random content each. We evaluate 4 different schedules ${ }^{4}$, which show an overhead between $3 \%$ and $10 \%$ (with Opt2). Hence, IVRs can perform much better than input-covering schedules (60\% overhead reported in [7]).

${ }^{4}$ As IMPARA cannot handle several features used by pfscan (such as condition variables, structs, and standard output), we manually generate initial IVRs. 


\section{Related Work}

Unbounded model checking $[18,20,32,39]$ is a technique to verify the correctness of potentially non-terminating programs. In our setting, we deploy algorithms that use abstract reachability trees (ARTs) $[21,28,39]$ to represent the already explored state space and schedules, and perform this exploration in a forward manner. Instead of discarding an ART after an unsuccessful attempt to verify a program, we use the ART to extract safe schedules.

Conditional model checking [8] reuses arbitrary intermediate verification results. In contrast to our approach, they are not guaranteed to prove the safety of a program that is functional under all inputs and does not enforce the preconditions (e.g., scheduling constraints) of the intermediate result.

Context bounding $[31,35,36]$ eases the model checking problem by bounding the number of context switches. It is limited to finite executions and unlike our approach, does not enforce schedules at runtime.

Automated fence insertion $[2,3,13,24,26]$ transforms a program that is safe under sequential consistency to a program that is also safe under weaker memory models. While the amount of non-determinism in the ordering of events is reduced, non-determinism due to scheduling can not be influenced. Synchronization synthesis [19] inserts synchronization primitives in order to prevent incorrect executions, but may introduce deadlocks.

Deterministic multi-threading (DMT) [4,6,7,11,12,27,30,34] reduces nondeterminism due to scheduling in multi-threaded programs. Schedules are chosen dynamically, depending on the explicit input, and can not be enforced by a model checker. Nevertheless, there are combinations with model checking [11] and instances which schedule based on previously recorded executions [12].

We are aware of only one DMT approach that supports symbolic inputs [7]. Similar to our sections, bounded epochs describe infinite schedules as permutations of finite schedules. Via symbolic execution, an input-covering set of schedules is generated, which contains a schedule for each permutation of bounded epochs. As all permutations need to be analyzed (even if they are infeasible), state space explosion through concurrency is only partially avoided; indeed, the experimental evaluation shows that the analysis is infeasible even for five threads when the program has many such permutations. In contrast, we do not require race-freedom, use model checking, sections may contain multiple threads, omit infeasible schedules, and allow a safe execution from the first schedule on, i.e., an IVR can be considerably smaller than an input-covering set of schedules.

Deterministic concurrency requires a program to be deterministic regardless of scheduling. In [37], a deterministic variant of a concurrent program is synthesized based on constraints on conflicts learned by abstract interpretation. In contrast to DMT, symbolic inputs are supported, however no verification of general safety properties is done and the degree of non-determinism is not adjustable, in contrast to IVRs. 
Sequentialized programs $[14,22,25,32,33,36]$ emulate the semantics of a multi-threaded program, allowing tools for sequential programs to be used. The amount of possible schedules is either not reduced at all or similar to context bounding.

\section{Conclusion}

We present a formal framework for using IVRs to extract safe schedules. We state why it is legitimate to constrain scheduling (in contrast to inputs) and formulate general requirements on model checkers in our framework. We instantiate our framework with the IMPACT model checking algorithm and find in our evaluation that it can be used to 1 . model check programs that are intractable for monolithic model checkers, 2. safely execute a program, given an IVR, even if there exist unsafe executions, 3. synthesize synchronization via assume statements, and 4 . guarantee fair executions. A drawback of enforcing IVRs is a potential execution time overhead, however, in several cases, constrained executions turned out to be even faster than unconstrained executions.

\section{References}

1. Benchmark suite of the competition on software verification (SV-COMP). https:// github.com/sosy-lab/sv-benchmarks

2. Abdulla, P.A., Atig, M.F., Chen, Y.-F., Leonardsson, C., Rezine, A.: Counterexample guided fence insertion under TSO. In: Flanagan, C., König, B. (eds.) TACAS 2012. LNCS, vol. 7214, pp. 204-219. Springer, Heidelberg (2012). https:// doi.org/10.1007/978-3-642-28756-5_15

3. Abdulla, P.A., Atig, M.F., Chen, Y.-F., Leonardsson, C., Rezine, A.: Memorax, a precise and sound tool for automatic fence insertion under TSO. In: Piterman, N., Smolka, S.A. (eds.) TACAS 2013. LNCS, vol. 7795, pp. 530-536. Springer, Heidelberg (2013). https://doi.org/10.1007/978-3-642-36742-7_37

4. Aviram, A., Weng, S., Hu, S., Ford, B.: Efficient system-enforced deterministic parallelism. In: OSDI. USENIX Association (2010)

5. Baier, C., Katoen, J.P.: Principles of Model Checking. MIT Press, Cambridge (2008)

6. Bergan, T., Anderson, O., Devietti, J., Ceze, L., Grossman, D.: CoreDet: a compiler and runtime system for deterministic multithreaded execution. In: ASPLOS. ACM (2010)

7. Bergan, T., Ceze, L., Grossman, D.: Input-covering schedules for multithreaded programs. In: OOPSLA (2013)

8. Beyer, D., Henzinger, T.A., Keremoglu, M.E., Wendler, P.: Conditional model checking: a technique to pass information between verifiers. In: FSE. ACM (2012)

9. Beyer, D., Keremoglu, M.E.: CPACHECKER: a tool for configurable software verification. In: Gopalakrishnan, G., Qadeer, S. (eds.) CAV 2011. LNCS, vol. 6806, pp. 184-190. Springer, Heidelberg (2011). https://doi.org/10.1007/978-3-642-22110-1_ 16

10. Clarke, E.M., Grumberg, O., Minea, M., Peled, D.: State space reduction using partial order techniques. STTT 2(3), 279-287 (1999) 
11. Cui, H., et al.: Parrot: a practical runtime for deterministic, stable, and reliable threads. In: SOSP. ACM (2013)

12. Cui, H., Wu, J., Gallagher, J., Guo, H., Yang, J.: Efficient deterministic multithreading through schedule relaxation. In: SOSP. ACM (2011)

13. Fang, X., Lee, J., Midkiff, S.P.: Automatic fence insertion for shared memory multiprocessing. In: ICS. ACM (2003)

14. Fischer, B., Inverso, O., Parlato, G.: CSeq: a concurrency pre-processor for sequential C verification tools. In: ASE. IEEE (2013)

15. Flanagan, C., Freund, S.N., Qadeer, S.: Thread-modular verification for sharedmemory programs. In: Le Métayer, D. (ed.) ESOP 2002. LNCS, vol. 2305, pp. 262-277. Springer, Heidelberg (2002). https://doi.org/10.1007/3-540-45927-8_19

16. Flanagan, C., Godefroid, P.: Dynamic partial-order reduction for model checking software. In: POPL. ACM (2005)

17. Godefroid, P. (ed.): Partial-Order Methods for the Verification of Concurrent Systems - An Approach to the State-Explosion Problem. LNCS, vol. 1032. Springer, Heidelberg (1996). https://doi.org/10.1007/3-540-60761-7

18. Günther, H., Laarman, A., Sokolova, A., Weissenbacher, G.: Dynamic reductions for model checking concurrent software. In: Bouajjani, A., Monniaux, D. (eds.) VMCAI 2017. LNCS, vol. 10145, pp. 246-265. Springer, Cham (2017). https:// doi.org/10.1007/978-3-319-52234-0_14

19. Gupta, A., Henzinger, T.A., Radhakrishna, A., Samanta, R., Tarrach, T.: Succinct representation of concurrent trace sets. In: POPL. ACM (2015)

20. Henzinger, T.A., Jhala, R., Majumdar, R.: Race checking by context inference. In: PLDI. ACM (2004)

21. Henzinger, T.A., Jhala, R., Majumdar, R., Sutre, G.: Lazy abstraction. In: POPL, pp. 58-70. ACM (2002)

22. Inverso, O., Tomasco, E., Fischer, B., La Torre, S., Parlato, G.: Bounded model checking of multi-threaded $\mathrm{C}$ programs via lazy sequentialization. In: Biere, A., Bloem, R. (eds.) CAV 2014. LNCS, vol. 8559, pp. 585-602. Springer, Cham (2014). https://doi.org/10.1007/978-3-319-08867-9_39

23. Kroening, D., Weissenbacher, G.: Interpolation-based software verification with Wolverine. In: Gopalakrishnan, G., Qadeer, S. (eds.) CAV 2011. LNCS, vol. 6806, pp. 573-578. Springer, Heidelberg (2011). https://doi.org/10.1007/978-3642-22110-1_45

24. Kuperstein, M., Vechev, M.T., Yahav, E.: Automatic inference of memory fences. In: FMCAD. IEEE (2010)

25. Lal, A., Reps, T.W.: Reducing concurrent analysis under a context bound to sequential analysis. Formal Methods Syst. Des. 35(1), 73-97 (2009)

26. Linden, A., Wolper, P.: A verification-based approach to memory fence insertion in PSO memory systems. In: Piterman, N., Smolka, S.A. (eds.) TACAS 2013. LNCS, vol. 7795, pp. 339-353. Springer, Heidelberg (2013). https://doi.org/10.1007/9783-642-36742-7_24

27. Liu, T., Curtsinger, C., Berger, E.D.: DTHREADS: efficient deterministic multithreading. In: SOSP. ACM (2011)

28. McMillan, K.L.: Lazy abstraction with interpolants. In: Ball, T., Jones, R.B. (eds.) CAV 2006. LNCS, vol. 4144, pp. 123-136. Springer, Heidelberg (2006). https://doi. org/10.1007/11817963_14

29. Metzler, P., Saissi, H., Bokor, P., Suri, N.: Quick verification of concurrent programs by iteratively relaxed scheduling. In: ASE. IEEE Computer Society (2017) 
30. Mushtaq, H., Al-Ars, Z., Bertels, K.: DetLock: portable and efficient deterministic execution for shared memory multicore systems. In: High Performance Computing, Networking Storage and Analysis. IEEE (2012)

31. Musuvathi, M., Qadeer, S.: Iterative context bounding for systematic testing of multithreaded programs. In: PLDI. ACM (2007)

32. Nguyen, T.L., Fischer, B., La Torre, S., Parlato, G.: Lazy sequentialization for the safety verification of unbounded concurrent programs. In: Artho, C., Legay, A., Peled, D. (eds.) ATVA 2016. LNCS, vol. 9938, pp. 174-191. Springer, Cham (2016). https://doi.org/10.1007/978-3-319-46520-3_12

33. Nguyen, T.L., Schrammel, P., Fischer, B., La Torre, S., Parlato, G.: Parallel bugfinding in concurrent programs via reduced interleaving instances. In: ASE. IEEE Computer Society (2017)

34. Olszewski, M., Ansel, J., Amarasinghe, S.P.: Kendo: efficient deterministic multithreading in software. In: ASPLOS (2009)

35. Qadeer, S., Rehof, J.: Context-bounded model checking of concurrent software. In: Halbwachs, N., Zuck, L.D. (eds.) TACAS 2005. LNCS, vol. 3440, pp. 93-107. Springer, Heidelberg (2005). https://doi.org/10.1007/978-3-540-31980-1_7

36. Qadeer, S., Wu, D.: KISS: keep it simple and sequential. In: PLDI. ACM (2004)

37. Raychev, V., Vechev, M., Yahav, E.: Automatic synthesis of deterministic concurrency. In: Logozzo, F., Fähndrich, M. (eds.) SAS 2013. LNCS, vol. 7935, pp. 283-303. Springer, Heidelberg (2013). https://doi.org/10.1007/978-3-642-38856-9_ 16

38. Valmari, A.: The state explosion problem. In: Reisig, W., Rozenberg, G. (eds.) ACPN 1996. LNCS, vol. 1491, pp. 429-528. Springer, Heidelberg (1998). https:// doi.org/10.1007/3-540-65306-6_21

39. Wachter, B., Kroening, D., Ouaknine, J.: Verifying multi-threaded software with impact. In: FMCAD. IEEE (2013) 